Boise State University ScholarWorks

IT and Supply Chain Management Faculty Department of Information Technology and Supply Publications and Presentations Chain Management

$1-1-1992$

\title{
Toward a Parsimonious Architecture for Intelligent Organizational Information Systems
}

Robert P. Minch

Boise State University 


\title{
Toward a Parsimonious Architecture for Intelligent Organizational Information Systems
}

\author{
Robert P. Minch \\ College of Business \\ Boise State University \\ Boise, ID 83725 \\ RISMINCH.IDBSU.EDU
}

\begin{abstract}
An architecture for intelligent organizational information systems is proposed which consists of three functions: processing, communicating, and memory--any or all of which may be performed by either humans or computers. Processing occurs on a set of communicating processors with access to memory, and is defined as having three sub-functions: sensing, interpreting, and acting. The communicating and memory functions are seen to have certain basic characteristics whether described in terms from human organization or computer organization literature. The architecture may prove a useful guide for future research which begins to consider intelligent organizational information systems with increasingly synergistic roles played by humans and computers.
\end{abstract}

\section{Introduction}

The solution to a problem should be as simple as possible, but no simpler --attributed to Einstein

When new technologies or applications of technologies emerge which are not adequately described by existing theory, researchers often propose frameworks, taxonomies, and other paradigms as media for discourse and further research. For example, the frameworks provided in [5] and [24] helped to guide research and practice in decision support systems. The present research seeks to outline an overall architecture for intelligent organizational information systems (OIS).

One goal of the architecture is to balance three important objectives of a framework dealing with manmachine systems:

1. Generality. It should be sufficiently abstract and general to incorporate the appropriate current and foreseeable technologies and organizational requirements, serving as a guide for current and future research.

2. Relevance. It should be sufficiently concrete and relevant to be of real usefulness to practitioners who design, build, use, and manage the systems of interest.

3. Parsimony. It should be concise and parsimonious, having the fewest number of elements and relationships between elements which adequately describe the model.

A second goal of the architecture is to begin to recognize the commonalities between other frameworks from various sources and investigate the benefits of their synthesis. It is not the intent here to minimize the importance of previously suggested frameworks or architectures for information systems. Indeed, they have often served as valuable guides for research and practice.

If a synthesis of multiple frameworks is possible, the benefits are several. Perhaps most importantly, it promotes more coordinated multi-disciplinary research efforts in the future by linking existing work into a common framework. This helps to make researchers in one area more aware of efforts from related areas in other disciplines, and serves to better define and focus research having similar goals. Secondarily, the synthesis may help to establish a more effective forum for discourse with a more concise vocabulary yet applying to a wider variety of contexts.

Organizational computing research draws on many diverse fields of study, including mathematics, computer science, organization science, information systems, economics, operations management, communications, linguistics, law, social psychology, and sociology [2]. Much of the previous research on OIS has relied on psychological, social-psychological, and organizational literature (e.g., [11]). While not attempting to equally represent all other potentially relevant disciplines, the

0073-1129-1/92 \$3.00 @ 1992 IEEE 
present research emphasizes concepts from computer science-related disciplines of data communications, computer system organization and design, and artificial intelligence. These perspectives will address the same basic characteristics of all information systems, which are related to capacities for processing, communication, and storage (memory) [12]. Many of the concepts taken from the different disciplines are remarkably similar in principle, differing in some cases primarily in nomenclature. Identification of important similarities across discipline-oriented perspectives will be addressed. The architecture is consistent with research in both human and computer organizations, and will provide integrative constructs in some areas.

The proposed architecture builds upon previous work [17] which discussed knowledge management in intelligent organizations and proposed hypermedia as a viable implementation vehicle. Hypermedia systems are able to store various forms of structured data, text, audio, and video information in typed nodes. Relating these nodes are typed links indicating relationships between nodes, including such relationships as one text node supporting another in an argumentative context, one program module being a subroutine of another, or one collection of data providing a more detailed representation of a summary datum. In multi-participant and organizational contexts, the flexible knowledge storage, organization, and retrieval mechanisms of hypermedia provide a valuable organizational associative memory. While knowledge management forms a critical part of the memory function for intelligent organizations, organizational information systems must also have processing and communicating functions. The present paper addresses all three components in an integrated framework.

Following sections will outline the proposed architecture and the three main functional components: processing, communications, and memory. The differing contributions of humans and computers will be addressed in each section. Then the special considerations involved in imbuing the architecture with intelligent characteristics will be discussed. Finally, a summary and conclusion section completes the paper.

\section{Basic architecture}

This section introduces the basic architecture, each component of which will be discussed further in later sections. There are three basic functions included in the architecture: processing, communication, and memory, defined as the following: Processing is the transformation of one or more inputs to one or more distinct outputs. It is accomplished by a processor or set of processors, each of which may be capable of executing several types of processes. Communication is the transmission of one or more output messages from one or more processes and receipt as input by one or more other processes. Memory is a buffer which facilitates communication by: (1) allowing transmission and receipt to occur asynchronously; (2) allowing multiple message receipts (storage re-accesses) from a single transmission; and (3) allowing message receipts in modes different from transmission mode (i.e., sequential storage but direct access).

There are three sub-functions involved in processing: sensing, interpreting, and acting. Sensing gleans data from internal sources or the physical world; interpretation creates information from the data; and acting allows information-based (and thus goal-driven) manipulation of internal memory and the physical world. The functions of processing, communicating, and memory form the basis from which more complex mechanisms may be constructed. Learning, for example, may involve a cycle of acting, followed by sensing the results, interpretation of the consequences, changes in memory, and perhaps a subsequent adaptive action. Overall system behavior exists in the context of communicating human and computer controlled processes with individual and shared goals and memory.

Similar architectures have been proposed by a number of other researchers. Newell [19] proposed a "general schema of a problem solver" which explicitly included processing and memory (both internal general knowledge and method stores) components, but emphasized a uniprocessor model where inter-processor communication was not critical. Processes included recognition of problems and information acquisition from the task environment (sensing), evaluation and internal representation (interpretation), and effectors which operate on the task environment (acting). The human problem solving model of Newell and Simon [20] includes a perceptual subsystem (sensing), cognitive subsystem (interpretation) including memory, and motor subsystem (acting). The essence of the standard top level in the LISP programming language--the so-called read-eval-print loop--involves reading (sensing), evaluating (interpreting) and printing (acting) [33]. Ackoff [1] described a "responsive decision system capable of rapid and effective learning and adaptation" including decision makers (processing), memory and information subsystems (memory), and flows in and out of an information subsystem (communication), but again in a uni-processor model. Processes were a surveillance function (sensing), diagnostic and prescriptive function (interpretation), and instructions sent to a controlled system (acting). Hall [10] defined a "process model of the natural logic of policy making," emphasizing processes such as an attention 
directing framework (sensing), perceiving and interpreting framework (interpretation), and enactment process (immediate precursor to action). Memory and communication among processing organizational subunits are recognized as components of this model.

For the architecture proposed here, reasonable scope of thesis and space limitations for the paper itself dictate a concentration on one level of analysis. This level is chosen to view the OIS as a communicating network of quasi-independent processors, each with their own memory. With the appropriate individual characteristics and communicating/coordinating mechanisms (discussed later), this system may exhibit behavior worthy of being called an intelligent organizational information system. Neither finer/lower levels of analysis (such as Newell's various method-related aspects of interpretation and acting [19]) nor coarser/higher levels of analysis (such as Hall's organizational-level driving forces [10]) will receive the focus of attention. Thus the next sections begin by discussing the basic three components of the architecture: processing, communication, and memory.

\section{Processing}

At the heart of the proposed architecture are generic processing functions which accept one or more inputs and produce one or more outputs. The set of four possible inputs and outputs considered is presented in Table 1 , along with the sixteen possible paired combinations. From these sixteen input/output dyads we can eliminate thirteen from consideration for the following reasons:

1. Four dyads have identical input and output types, which is regarded in this architecture as inconsistent with the definition of a process as a mechanism for transforming an input into a distinct output. For example, data is processed to produce information. This information may be used as data for another process, but we ignore the degenerative case where a process has no effect on its own input.

2. Four dyads imply functions which are the inverse of those defined in the architecture. Order of input and output is significant, so for example physical stimuli may produce data but data may not (directly) produce physical stimuli.

3. Four dyads imply multiple processes to produce the output from the input, whereas the architecture considers atomic processes individually. For example, physical action is not considered to be a (direct) output from a data input because an intermediary process (processing data into information) is required. This particular example has intuitive appeal in that it requires sentient behavior to produce action.
4. Finally, one dyad describes an implicit process (physical action leading to physical stimuli), which may or may not occur but is considered to be outside the boundaries of the system under investigation. An example would be a process which produces some action but, before the results of that action are sensed, an intervening change such as physical decay occurs.

The three remaining inputs/output dyads define the process portion of the proposed architecture, and are shown in Figure 1. This figure shows the three basic forms of processing relevant to organizational information systems: (1) data is captured from internal sources or the physical world through a sensing process; (2) information is created from data in an interpretation process; and (3) information is used to update memory or manipulate the physical world through an acting process. Other mechanisms which transform one state of the physical world to another, while certainly occurring, are outside the architecture system's boundaries (although the results of these transformations may be detected by sensing processes). Inputs to all processes may originate inside or outside the organization, with outputs similarly having effect internally or externally. The sequential, closed loop of linked processes shown in Figure 1 does not imply any specific source of particular inputs, destination of particular outputs, or time-phasing of inter-process communication.

Processors (vehicles for accomplishing processing) may be one of several types, the principal types of interest to organizational information systems being humans and computers. With the advent of computerized systems exhibiting sufficient intelligence to be indistinguishable from human intelligence and pass the Turing test [29] in limited domains, it is now important to consider organizations consisting of both human and computerized processors. Already the complementary roles of humans and computers have been addressed in the context of systems such as management support systems. It is suggested, for instance, that in decision support systems the human queries the machine, while in expert systems the machine queries the human [28].

Many processes may be executed by one processor, either through serial processing (each process is completed before the next begins), multi-programming (concurrent but not simultaneous processing of several processes over the same general time period though time sharing), or parallel operations (processors which can accomplish multiple simultaneous transformations, as in vector arithmetic units). A process may involve subprocesses which must be completed for successful process completion. Of course processes may also be combined to achieve higher level goals. 
Control of processes may take several forms, including centralized and decentralized control. Agency relationships may be established where one process serves or acts on behalf of another process. The following paragraphs discuss the three basic processes.

\subsection{Sensing}

Human senses have traditionally formed the primary source of input to information systems in organizations. All five human senses can also be found, however, with varying degrees of sensitivity, in automated or computerized form [23]. In many cases machine sensing is much more accurate than its human counterpart (for instance, temperature measurement), while in others it greatly extends the range of detectable and measurable inputs (for example, observation of electromagnetic radiation). A database of sensor types under development is said to anticipate some 30,000 items [23].

Future OIS striving for intelligent behavior will need to exploit both human and automated sensory capabilities where each is best suited. For example, in the foreseeable future humans will probably retain their advantage in sensing complex audio and visual stimuli such as voice inflections and facial expressions. Machines, however, often perform better than humans when filtering structured data and text according to welldefined criteria--such as searching stock market data for potentially profitable arbitrage opportunities.

\subsection{Interpreting}

The core process of information systems, interpreting involves processing data from a variety of internal and external sources and transforming it into information. This information reduces uncertainty and may be used as an input to subsequent processing or action. One source of data may be sensors, while another may be the output from other interpreting processes (since interpretation may be an iterative or even a recursive process). Data from internal sources is critical for planning and control, while that from external sources is an important input for environmental scanning.

Environmental scanning is a particularly significant and increasingly important activity undertaken by intelligent organizations. Executive requests for information found outside their company's databases are increasing at an average rate of $18 \%$ to $19 \%$ per year [27], while users of corporate libraries find external electronic database services more valuable than other sources of information such as journals, books, and syndicated studies [21]. There are over 300 subscription document retrieval systems in operation [8]. To be effectively used, external data must be transformed into a useful form through techniques such as filtering, reformatting, restructuring, and indexing. Conversion to a form more amenable to knowledge-based processing, such as cognitive graphs, has also been proposed [6].

Traditionally, interpretation has been the domain of human processors. Recognition of patterns and trends in data, forming hypotheses and theories, and generalpurpose decision making/problem solving were generally acknowledged to require uniquely human traits such as intelligence. Of course, the significant strides in the field of artificial intelligence have now successfully been applied to many of these areas, significantly increasing the viability of computers as interpreting processors. Just as computer networks today employ file servers and print servers to share valuable scarce resources, future networked OIS may employ knowledge servers and expert servers (both human and computerized) which serve clients by sharing scarce expertise and decision making abilities organization-wide in response to service requests. Valuable human-computer synergistic approaches already include human-directed computerized agents or daemons [16] which search electronic mail and other databases according to rules designed to retrieve and process pertinent information.

\subsection{Acting}

As with sensing, traditional information systems rely heavily on human components to carry out necessary actions deemed appropriate. Information created from the interpretation and decision making processes normally require manipulation of the physical world to implement those decisions. Two areas of advancement have led to an increasing role of machines in acting, however. First, computerized processors (including those employing artificial intelligence) may affect informational changes in the state of system components to which they are connected. Thus they may delete, modify, or add to system memory. Second, the advent of numerically controlled machines and robotics gives machines the means to take physical actions. Computer-controlled actors, particularly those with sensors providing feedback to facilitate goal-directed behavior, will be important to future intelligent OIS.

\section{Communication}

Table 2 shows communications characteristics and their interpretations for computer-computer communications (synthesized from [25]) in the first two columns, and a more general interpretation for organizational communication (including the four message processing 
activities from [11]) in the third column. From examining the table, it is obvious that basic communication methods, modes, and protocols are remarkably similar regardless of whether the communicating entities are humans or computers. For example, all messages must be encoded for transmission, whether it be in a computerized coding scheme such as ASCII or a human medium of exchange such as spoken or written language.

The most important differences between the computer communication and human communication perspectives probably lie not in the technical aspects such as protocols, but rather in the reasons for communication and effects of those communications. Organizational theorists maintain that organizations process information (and hence communicate) to reduce uncertainty [9] and reduce equivocality [32]. Computer communication, on the other hand, currently exists on a more rudimentary and structured level concerned primarily with such functions as terminal sessions, distributed database access, and electronic data interchange (for transactions processing). There are relationships between the higher-level goals and lower-level functions, however. Consider, for example, the finding that humans prefer face-to-face communication for messages containing equivocality, while written media is acceptable for unequivocal messages [15]. The high level communications methods (e.g., face-to-face versus written) often incorporate several lower level communications functions or characteristics. For instance, face-to-face human communication can be at least partially described in computer communications terminology as multi-modal (verbal supplemented by gestures, physical cues, etc.), full duplex, synchronous, high-bandwidth communication.

The movement toward more synergistic human and computer participation in intelligent OIS will lead to a greater variety of communications methods, borrowing from and exploiting the capabilities of each participant type. We can expect more computer-supported and computer-mediated human communication as well as more research into translation facilities, such as computerized natural language processing, which will help disparate entities communicate more effectively. We can also expect a more generalized implementation of both peer-topeer and client-server network coordination models. For example, humans may still request data from a file server, but computerized processors might also request cognitive services from human "intelligence" or "creative" servers.

\section{Memory}

Organizational memory exists in the context of sensing/interpreting organizational information systems [31]. Sensors receive data from internal or environmental sources, the interpreted information and knowledge is stored (symbolically, if it exists outside the human mind), and retrieval mechanisms provide subsequent access to the stored information and knowledge. Memory contents may be replaced or supplemented as new information becomes available or as processes such as re-interpretation or summarization are applied to existing contents.

In previous work hypermedia was proposed as a vehicle for knowledge management in OIS [17]. Hypermedia systems are computer-implemented networks of nodes (database or media objects) and links (arcs used to connect and traverse between nodes). Because nodes can be typed and may contain multimedia contents, they are appropriate for a wide variety of OIS memory, including free text prose, structured data, rules and other knowledge representations, mathematical models, algorithms, user-defined conceptual entities, quantitative and qualitative variables, and audio/visual information. Links may also be typed, and may be used to store semantically significant relationships between nodes such as cause/effect; dialectical, issue-based, or argumentative analysis; and formal logical/mathematical associations [7]. Mechanisms to provide longitudinal process memory over time can be incorporated into hypermedia. Learning is supported by and manifested in the ability of organizational entities to add, modify, and delete nodes and links. Knowledge management may be partially automated through mechanisms which semi-automatically combine sub-networks [14] and automatically create some links [4].

When we consider a system of communicating or networked processors (the term networked implying not only that communication is possible, but that it occurs on an ongoing basis in a relatively stable configuration), the structure of OIS memory becomes quite complex. Memory may be associated with individual processes and processors or may be shared by several processes and processors. So for example if we use hypermedia memory, each processor might have its own memory network, each with links to other processors' memory networks, forming a memory web with potentially great size and complexity. Not all processes may access all memory, however. This is a particularly binding constraint when processors of different types such as humans and computers wish to share memory. In this case the hurdles to be overcome include greatly different organization and access methods, typically requiring output from one memory system to be actionalized by one processor to an external form which may be sensed and re-interpreted by a second processor and stored in a memory system to which the second processor has direct access.

Table 3 summarizes important characteristics of 
memory in computer organization terms (column 2) and human/organizational terms (column 3). Of course the organization of the memory contents is itself highly variable, and is more closely associated with processes using the memory than the memory itself.

\section{Intelligence}

Human intelligence is a nebulous concept, perhaps best described in general terms of adaptability to changing environmental conditions. Non-human intelligence assumes a derivative definition, thus artificial intelligence may be defined as "the science of making machines do things that would require intelligence if done by men" [18]. Similarly, organizations which are said to be intelligent must exhibit an analogous level of adaptability as compared to human counterparts, albeit through possibly different mechanisms. A critical component of intelligence and adaptability is learning, discussed below.

\subsection{Learning}

Learning involves the creation and refinement of cognitive structures that serve as a basis for action [13], a process which in humans is not well understood and described primarily by its observable artifacts such as improved performance over time. Machine learning has been the subject of somewhat more precise (but not necessarily more useful) definition. One definition, for instance, states that machine learning attempts to achieve one or more of the following goals [8]:

1. Provide more accurate solutions

2. Cover a wider range of problems

3. Obtain answers more economically

4. Simplify codified knowledge

Multi-computer architectures for intelligent systems have been proposed in some fifteen distinct configurations [30], in which learning may be distributed among processors in various ways. These range from smallgrained connectionist or neural-network organizations with many semi-autonomous processors to larger-grained organizations where learning activities are assigned to a smaller number of separate computers. The same concepts may be extended to multi-process architectures including both human and computer processors. In this proposed intelligent OIS architecture, learning may be manifested in at least two ways. First, memory contents, linkages, or organization may be updated. Second, the performance of processing functions themselves may be improved (thus sensing, interpreting, and or acting may be refined, made more efficient, or made more effective).

It has been argued that the rate at which individuals and organizations learn may become the only sustainable competitive advantage, especially in knowledge-intensive industries [26]. Organizations must focus not only on adaptive/coping learning, but more creative generative learning [3], [22].

\section{Summary and conclusions}

The fledgling area of intelligent organizational information systems is built upon complex human organizations, computerized information systems, and the myriad interactions between the two. If we are to meld these components into an integrated whole, it is important to develop a consistent framework which both recognizes the unique characteristics of its differing components and the areas where they are similar. The proposed architecture portrays intelligent OIS as a set of communicating processes, each executing on a human or computerized processor and each with individual and/or shared memory, capabilities, and goals. It is not proposed that organizations of humans and organizations of computers need to be organized according to one monolithic structure. Rather, the growing complexity of human organizations and increasing capabilities of computerized systems leads us to consider unifying paradigms where the responsibilities of each member are more effectively and synergistically assigned and carried out, and correspondingly more effective and intelligent organizational behavior results.

\section{References}

[1] Ackoff, R. "Beyond Prediction and Preparation," Journal of Management Studies, Vol. 20, No. 1, 1983, pp. 59-69.

[2] Applegate, L., et al., "Organizational Computing: Definitions and Issues," Journal of Organizational Computing, Vol. 1, No. 1, January-March 1991, pp. $1-10$.

[3] Argyris, C. and Schon, D. Organizational Learning: A Theory-in-Action Perspective. Reading, Mass.: Addison-Wesley, 1978.

[4] Bhargava, H.; Bieber, M.; and Kimbrough, S. Oona, Max, and the WYWWYWI principle: Generalized hypertext and model management in a symbolic programming environment. Proceedings of the Ninth International Conference on Information Systems, Minneapolis, (December 1988), 179-191.

[5] Bonczek, R. H.; Holsapple, C. W.; and Whinston, A. B. Foundations of Decision Support Systems. New York: Academic Press, 1981. 
Bonham, G. M. et al. "Cognitive Graphing and the Representation of Biomedical Knowledge," Proceedings of the Expert Systems in Government Conference, IEEE Computer Society Press, 1985.

Conklin, F. A Survey of Hypertext. Microelectronics and Computer Technology Corporation, MCC Technical Report Number STP-356-86, 1987.

Forsyth, R. and Rada, R. Machine Learning: Applications in Expert Systems and Information Retrieval. New York: Halstead Press, 1986.

Galbraith, J. Designing Complex Organizations, Reading, Mass.: Addison-Wesley, 1973.

[10] Hall, R. "The Natural Logic of Management Policy Making: It's Implications for the Survival of an Organization," Management Science, Vol. 30, No. 8, August 1984, pp. 905-927.

[11] Huber, G. "Organizational Information Systems: Determinants of their Performance and Behavior," Management Science, Vol. 28, No. 2, February 1982, pp. 138-155.

[12] Huber, G. P. "A Theory of the Effects of Advanced Information Technologies on Organizational Design, Intelligence, and Decision Making," Academy of Management Review, Vol. 15, No. 1, 1990, pp. 4771.

[13] Hunt, R. G. and Sanders, G. L. "Supporting Intelligent Organizations: Problem Solving and Learning," Proceedings of the Twenty-Second Annual Hawaii International Conference of System Sciences (Vol. III), IEEE Press, 1989, pp. 476-482.

[14] Lee, S. and Courtney, J. Organizational learning systems. Proceedings of the Twenty-Second Annual Hawaii International Conference on System Sciences, Vol. III, Kona, Hawaii, (January 1989), 492-503.

[15] Lengel, R. H. and Daft, R. L. "An Exploratory Analysis of the Relationship Between Media Richness and Managerial Information Processing," Working Paper, Texas A \& M University, 1984.

[16] Malone, T.; Grant, K. R.; Turbak, F. A.; Brobst, S. A.; and Cohen, M. D. Intelligent information sharing systems. Communications of the ACM, 30, 5 (May 1987), 390-402.

[17] Minch, R. P. "Hypermedia Knowledge Management for Intelligent Organizations," Proceedings of the Twenty-Third Annual Hawaii International Conference on System Sciences (Vol. IV), IEEE Computer Society Press, 1990, pp. 300-306.
[18] Minsky, M. L. (ed.) Semantic Information Processing. Cambridge, Mass: MIT Press, 1968.

[19] Newell, A. "Heuristic Programming: Ill-Structured Problems," in Publications in Operations Research No. 16 (ed. J. Aronofsky). New York: John Wiley \& Sons, 1969, pp. 361-414.

[20] Newell, A. and Simon, H. Human Problem Solving. Englewood Cliffs, NJ: Prentice-Hall, 1972.

[21] O'Connel, L. "Extending Walls of Corporate Libraries," Computerworld, June 25, 1990, p. 81.

[22] Senge, P. M. "The Leader's New Work: Building Learning Organizations," Sloan Management Review, Vol. 32, No. 1, Fall 1990, pp. 7-23.

[23] Simons, G. Eco-Computer: The Impact of Global Intelligence. New York: John Wiley \& Sons, 1987.

[24] Sprague, R. and Carlson, E. Building Effective Decision Support Systems. Englewood Cliffs, NJ: Prentice-Hall, 1982.

[25] Stallings, W. Business Data Communications. New York, Macmillan, 1990.

[26] Stata, R. "Organizational Learning: The Key to Management Innovation," Sloan Management Review, Vol. 30, No. 3, Spring 1989, pp. 63-74.

[27] Stevens, L. "Channeling the Flood of Data from Outside On-line Services," Computerworld, June 25, 1990, pp. 80-81.

[28] Turban, E. Decision Support and Expert Systems. New York: Macmillan, 1990.

[29] Turing, A.M. "Computing Machinery and Intelligence," Mind, Volume 59, 1950, p. 236.

[30] Uhr, L. Multi-Computer Architectures for Artificial Intelligence. New York: John Wiley \& Sons, 1987.

[31] Walsh, J. P. "Organizational Memory," Academy of Management Review, Vol 16, No. 1, 1991, pp. 57-91.

[32] Weick, K. E. The Social Psychology of Organizing. Reading, Mass.: Addison-Wesley, 1979.

[33] Wilenski, R. LISPcraft. New York: W. W. Norton, 1984. 
Table 1: Process Input/Output Combinations

\begin{tabular}{|c|c|c|c|c|c|}
\hline & & \multicolumn{4}{|c|}{ Outputs } \\
\hline & & Physical stimuli & Data & Information & Physical action \\
\hline \multirow{4}{*}{ Inputs } & $\begin{array}{l}\text { Physical } \\
\text { stimuli }\end{array}$ & $\begin{array}{l}\text { N/A: input \& } \\
\text { output not } \\
\text { distinct }\end{array}$ & Sensing & $\begin{array}{l}\text { N/A: requires } \\
\text { intermediate } \\
\text { processing }\end{array}$ & $\begin{array}{l}\text { N/A: } \\
\text { definitional } \\
\text { inverse }\end{array}$ \\
\hline & Data & $\begin{array}{l}\text { N/A: definitional } \\
\text { inverse }\end{array}$ & $\begin{array}{l}\text { N/A: input \& } \\
\text { output not } \\
\text { distinct }\end{array}$ & Interpreting & $\begin{array}{l}\text { N/A: requires } \\
\text { intermediate } \\
\text { processing }\end{array}$ \\
\hline & Information & $\begin{array}{l}\text { N/A: requires } \\
\text { intermediate } \\
\text { processing }\end{array}$ & $\begin{array}{l}\text { N/A: } \\
\text { definitional } \\
\text { inverse }\end{array}$ & $\begin{array}{l}\text { N/A: input \& } \\
\text { output not } \\
\text { distinct }\end{array}$ & Acting \\
\hline & $\begin{array}{l}\text { Physical } \\
\text { action }\end{array}$ & Implicit process & $\begin{array}{l}\text { N/A: requires } \\
\text { intermediate } \\
\text { processing }\end{array}$ & $\begin{array}{l}\text { N/A: } \\
\text { definitional } \\
\text { inverse }\end{array}$ & $\begin{array}{l}\text { N/A: input \& } \\
\text { output not } \\
\text { distinct }\end{array}$ \\
\hline
\end{tabular}

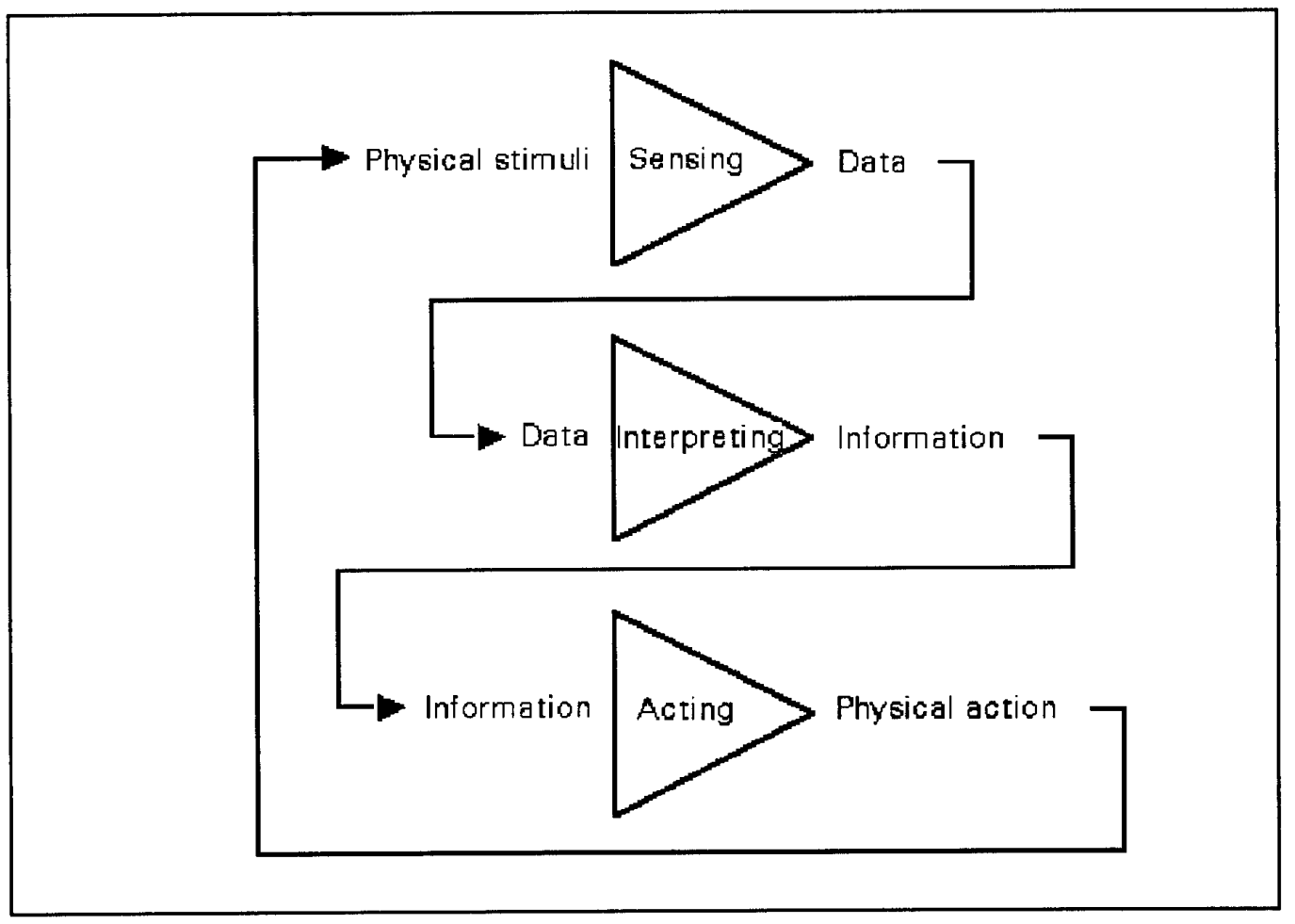

Figure 1: Basic Process Types 
Table 2: Communication Characteristics

\begin{tabular}{|c|c|c|}
\hline Communications Characteristic & Computer-computer communication & Organizational communication \\
\hline Media & Electrical, optical conductors & $\begin{array}{l}\text { Information carrier: paper, computer, } \\
\text { audio, video }\end{array}$ \\
\hline Media ownership & Private, common carrier & $\begin{array}{l}\text { Individual, intraorganizational, } \\
\text { organizational, inter-organizational, } \\
\text { public }\end{array}$ \\
\hline Duplex & Simplex, half-duplex, full duplex & $\begin{array}{l}\text { Monologue, dialogue, concurrent } \\
\text { dialogue }\end{array}$ \\
\hline Coding techniques & $\begin{array}{l}\text { ASCII, EBCDIC, etc.; data compression } \\
\text { techniques }\end{array}$ & Language, message summarizing \\
\hline Coordination with respect to time & Asynchronous, synchronous & Different time, same time \\
\hline Geographical area & Local, metropolitan, wide area network & Same location, dispersed locations \\
\hline Bandwidth & Maximum signalling and transmission rate & $\begin{array}{l}\text { Information transmission and } \\
\text { assimilation rate, message delay }\end{array}$ \\
\hline Signalling mode & Analog, digital & Text, graphics, audio, video \\
\hline Receiver identification & Point-to-point, multicast, broadcast & Private, group, general announcements \\
\hline Signal/noise ratio & Low-level signalling efficiency & $\begin{array}{l}\text { Communications effectiveness, message } \\
\text { modification }\end{array}$ \\
\hline Switching method & Circuit, message, packet & Message routing \\
\hline Multiplexing method & Time division, frequency division & Multi-channel control \\
\hline Reliability & Mean time between failures & Probability of message receipt \\
\hline Security & $\begin{array}{l}\text { Cost, time, difficulty, probability of } \\
\text { breach }\end{array}$ & $\begin{array}{l}\text { Same as computer-computer } \\
\text { communication plus issues of privacy }\end{array}$ \\
\hline Cost effectiveness & $\begin{array}{l}\text { Quantity or value of throughput per unit } \\
\text { cost }\end{array}$ & Value of information communicated \\
\hline Network topology & Bus, star, ring, hybrid & $\begin{array}{l}\text { Links between departments, groups, } \\
\text { project teams, etc. }\end{array}$ \\
\hline Network access protocols & $\begin{array}{l}\text { OSI layers } 1,2 \text {, and } 3 \text {, including } \\
\text { electrical, optical, and mechanical signal } \\
\text { characteristics }\end{array}$ & $\begin{array}{l}\text { Basic communications method used: } \\
\text { vocalization, writing, etc. }\end{array}$ \\
\hline Transport protocols & $\begin{array}{l}\text { OSI layer } 4 \text {, providing reliable end-to-end } \\
\text { communication, controlling syntax, } \\
\text { semantics, and timing }\end{array}$ & Conversational etiquette \\
\hline Application protocols & $\begin{array}{l}\text { OSI layers } 5,6 \text {, and } 7 \text {, including session } \\
\text { management, data transformation, and } \\
\text { user-level applications }\end{array}$ & $\begin{array}{l}\text { Meeting management, organizational } \\
\text { communication channels and policies }\end{array}$ \\
\hline
\end{tabular}


Table 3: Memory Characteristics

\begin{tabular}{|l|l|l||}
\hline Memory characteristic & Computer organization & Human organization \\
\hline \hline Volatility & $\begin{array}{l}\text { Volatile (e.g., DRAM), non-volatile } \\
\text { (e.g., magnetic disk) }\end{array}$ & $\begin{array}{l}\text { Relatively temporal to relatively } \\
\text { permanent or institutionalized }\end{array}$ \\
\hline Access speed & $\begin{array}{l}\text { Nanosecond (e.g., register) through } \\
\text { millisecond (e.g., magnetic disk) for } \\
\text { on-line storage }\end{array}$ & $\begin{array}{l}\text { Millisecond (short term human } \\
\text { memory), multi-second (some long } \\
\text { term memory), longer for } \\
\text { organization-wide access }\end{array}$ \\
\hline Access mode & Sequential, direct & $\begin{array}{l}\text { Direct if on-line (human internal } \\
\text { memory), possibly sequential if off- } \\
\text { line (external memory) }\end{array}$ \\
\hline Access direction & Read-only, read-write & $\begin{array}{l}\text { Read-only for some external } \\
\text { memory, read-write for human } \\
\text { internal memory }\end{array}$ \\
\hline Accessibility & On-line, off-line & $\begin{array}{l}\text { On-line for human internal memory, } \\
\text { off-line for some secondary, } \\
\text { external, or organization-wide } \\
\text { memory }\end{array}$ \\
\hline Cost & Varies by capacity and speed & $\begin{array}{l}\text { External memory cost varies by } \\
\text { capacity and speed }\end{array}$ \\
\hline
\end{tabular}

